\title{
Efficacy of Modified Expansive Open-Door Laminoplasty for Cervical Myelopathy
}

\author{
Eiren Toh $^{1}$, Yukihiro Yamamoto ${ }^{2}$, Daisuke Sakai ${ }^{2}$, Masato Sato ${ }^{2}$, Masahiko Watanabe $^{2}$, Joji Mochida $^{2}$ \\ ${ }^{1}$ Department of Orthopaedic Surgery, Tokai University Hachioji Hospital, Tokyo, Japan \\ ${ }^{2}$ Department of Orthopaedic Surgery, Tokai University School of Medicine, Kanagawa, Japan \\ Email: eiren@is.icc.u-tokai.ac.jp
}

Received October 2, 2012; revised November 4, 2012; accepted November 14, 2012

\begin{abstract}
Objectives: Expansive open-door laminoplasty is used widely for the treatment of cervical spondylosis and Ossification of the Posterior Longitudinal Ligament (OPLL). We have developed a unique modification of the surgical procedure to keep the lamina expanded, with the aim of preventing reclosure of the vertebral arch. To examine the effectiveness of and problems associated with the modified expansive open-door laminoplasty technique developed at our institution by evaluating the surgical outcomes. Methods and Materials: Fifty-six patients (46 men and 10 women) underwent the modified expansive open-door laminoplasty and were followed up for at least 1 year. Thirty-eight had Cervical Spondylotic Myelopathy (CSM) and 18 had OPLL. The patients were 34 to 89 years of age (mean: 60.9 years). The severity of myelopathy was evaluated according to the Japanese Orthopaedic Association's scoring system. Surgical outcomes were evaluated using Hirabayashi's system for determining recovery rate. In the radiographic analysis, the following angles were measured before and after surgery: lordosis angle and Range of Motion (ROM) at C2 - C7 on lateral radiographs, and opening angle on Computerized Tomography (CT). The presence and absence of axial pain and postoperative C5 palsy were also evaluated. Results: The rate of JOA score improvement was about $60 \%$, the lordosis angle observed on lateral radiographs was maintained. ROM decreased after surgery in both the CSM and OPLL groups, and the extent of the decrease was similar to that in previous reports. The opening angle of the lamina was $62^{\circ}-65^{\circ}$ on postoperative CT. Axial pain was reported by $34 \%$ of patients. Conclusions: Our modified procedure produced satisfactory postoperative outcomes based on the clinical data and imaging findings for both CSM and OPLL. The advantage of this procedure is that it avoids potential complications associated with bone grafts or implants.
\end{abstract}

Keywords: Cervical Myelopathy; Surgical Treatment; Middle and Lower Cervical Spine

\section{Introduction}

Expansive open-door laminoplasty is used widely for the treatment of cervical spondylosis and Ossification of the Posterior Longitudinal Ligament (OPLL) and has been reported to have a marked decompression effect and to yield good clinical outcomes [1-3]. In the original procedure, the vertebral arch is fixed to the articular capsule at the hinge side by suturing to keep it expanded. A number of modified surgical procedures have been reported since, in which a spacer bone graft, bone substitutes, or miniplates are used to ensure that the lamina is kept in the opened position [4-11]. We have developed a unique modification of the surgical procedure to keep the lamina expanded, with the aim of preventing reclosure of the vertebral arch and the complications that may result from the emplacement of spacer bone grafts, bone substitutes, or miniplates.

\section{Objective}

In this study, we examined the effectiveness of and pro- blems associated with the modified expansive open-door laminoplasty technique developed at our institution by evaluating the surgical outcomes.

\section{Methods}

\subsection{Surgical Procedure}

In our modified surgical procedure, to keep the lamina expanded, a stay suture is passed through an opening in the base of the spinous process and lower interspinous ligaments, and the suture is then fixed on the fascia at the hinge side, $2 \mathrm{~cm}$ from the center. The most caudal part and lower part of the dilated spinous processes are tied by a figure-of-eight suture to prevent reclosure of the expanded vertebral arch (Figure 1) [12].

\subsection{Patients}

Fifty-six patients (46 men and 10 women) underwent the modified expansive open-door laminoplasty at our institution during the period 2001 to 2005 and were followed 
up for at least 1 year (Table 1). Thirty-eight had Cervical Spondylotic Myelopathy (CSM) and 18 had OPLL. The patients were 34 to 89 years of age (mean: 60.9 years).

\subsection{Evaluations}

The severity of myelopathy was evaluated according to the Japanese Orthopaedic Association (JOA) scoring system (Table 2). Surgical outcomes were evaluated using Hirabayashi's system for determining recovery rate. In the radiographic outcomes, the following angles were measured before and after the surgery: the lordosis angle

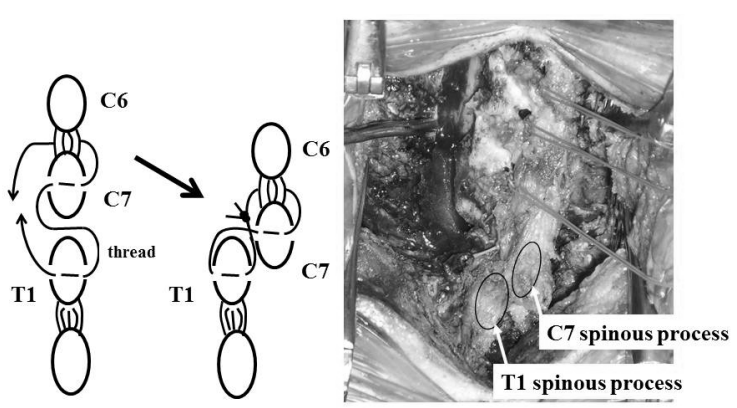

(a)

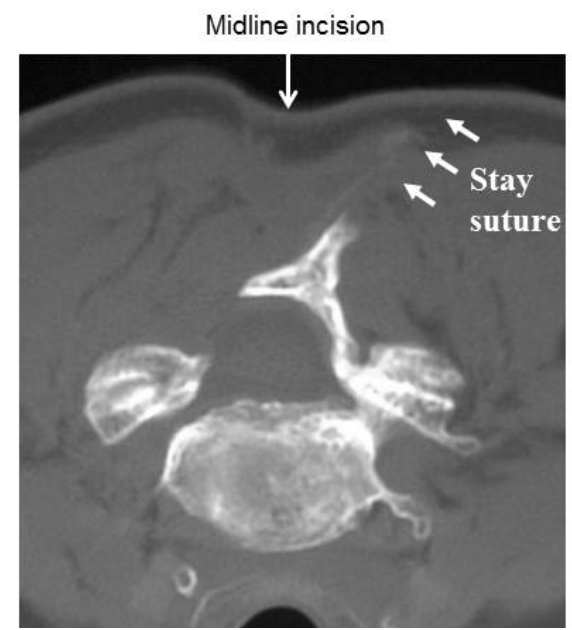

(b)

Figure 1. Surgical procedure. (a) Left: schematic drawing; right: perioperative photograph. Suture passed through the foramen is opened at the base of the spinous process and is fixed by a figure-of-eight suture in the recurvation position; (b) Axial CT image obtained 6 months after the surgery (C5). The stay suture is fixed on the fascia at the hinge side, $2 \mathrm{~cm}$ from the center.

Table 1. Patients’ demographics.

\begin{tabular}{ccc}
\hline & CSM & OPLL \\
\hline Number of patients & 38 & 18 \\
Number of males/females & $29 / 9$ & $17 / 1$ \\
Average age at the time & $60.9 \mathrm{ys}$ & $61.0 \mathrm{ys}$ \\
of the surgery (Range) & $(34-82)$ & $(49-73)$ \\
Average follow-up period & $2.9 \mathrm{ys}$ & $2.3 \mathrm{ys}$ \\
(Range) & $(1.0-6.0)$ & $(1.1-3.5)$ \\
\hline
\end{tabular}

and the range of motion (ROM) at C2 - C7 on lateral radiographs measured using Cobb's method, and the opening angle on Computerized Tomography (CT). The presence or absence of axial pain and postoperative C5 palsy were also evaluated. The data were analyzed using the Mann-Whitney $U$ test using the median values because of large variations in these values.

\section{Results}

The mean JOA score improved from before the procedure (10.3 for patients with CSM and 9.5 for patients with OPLL) to the last visit (14.3 and 14.1, respectively). The improvement rate was $59.6 \%$ for CSM and $61.7 \%$ for OPLL (Table 3). The mean preoperative JOA scores,

Table 2. Japanese Orthopaedic Association (JOA) scoring system for cervical myelopathy.

\begin{tabular}{ll}
\hline I. Motor function \\
\hline \multicolumn{2}{c}{ Upper extremity: feeding oneself with chopsticks or a spoon } \\
\hline (0) & Total disturbance: unable to feed oneself \\
(1) Severe disturbance: unable to use chopsticks \\
(2) Moderate disturbance in the use of chopsticks \\
(3) Mild disturbance in the use of chopsticks \\
(4) Normal
\end{tabular}

\begin{tabular}{ll}
\hline II. Motor function \\
\hline \multicolumn{2}{c}{ Lower extremity: gait } \\
\hline (0) & Total disturbance: unable to walk \\
$(1)$ & Severe disturbance: needs aid on flat ground \\
$(2)$ & Moderate disturbance: needs aid on stairs \\
$(3)$ & Mild disturbance: needs no aid on stairs but unstable \\
$(4)$ & Normal \\
\hline
\end{tabular}

\begin{tabular}{l}
\hline III. Sensory function \\
\multicolumn{2}{c}{ Upper extremity, lower extremity and trunk } \\
\hline (0) Complete or apparent sensory loss or severe paraesthe- \\
sia \\
(1) Mild sensory loss or paraesthesia \\
(2) Normal
\end{tabular}

IV. Bladder function

(0) Total disturbance: complete retention or complete incontinence

(1) Severe disturbance: incomplete retention, incomplete incontinence, or straining

(2) Moderate disturbance: frequency or hesitation

(3) Normal

Table 3. Result of JOA score.

\begin{tabular}{cccc}
\hline & CSM & OPLL & P \\
\hline Preoperative JOA & $10.3 \pm 2.05$ & $9.5 \pm 2.61$ & NS \\
score & $(6-13)$ & $(5-13)$ & \\
Postoperative JOA & $14.3 \pm 2.01$ & $14.1 \pm 1.93$ & NS \\
score & $(9-17)$ & $(11-17)$ & \\
Recovery Rate & 59.6 & 61.7 & NS \\
\hline
\end{tabular}

NS indicates not significant. 
postoperative JOA scores, and recovery rates did not differ significantly between patients with CSM and OPLL.

The lordosis angle measured on lateral radiographs before the procedure, 6 months after the procedure, and at the last observation were $12.7^{\circ}, 11.3^{\circ}$, and $10.5^{\circ}$, respectively, in the patients with CSM and $10.9^{\circ}, 8.2^{\circ}$, and $5.6^{\circ}$ in the patients with OPLL (Table 4). The mean preoperative and postoperative lordosis angles did not differ significantly between the CSM and OPLL groups.

ROM was measured before the procedure, 6 months after the procedure, and at the last observation. The ROM values were $31.5^{\circ}, 19.9^{\circ}$, and $20.6^{\circ}$ in the patients with CSM, and $29.5^{\circ}, 15.5^{\circ}$, and $14.0^{\circ}$ in the patients with OPLL (Table 5). The mean preoperative ROM did not differ significantly between the CSM and OPLL groups, but the mean postoperative ROM was significantly greater in the patients with CSM than in those with OPLL.

The opening angle measured on CT was $65.9^{\circ}$ for $\mathrm{C} 4$ and $64.6^{\circ}$ for C5 in the patients with CSM, and $63.5^{\circ}$ for $\mathrm{C} 4$ and $62.8^{\circ}$ for C5 in the patients with OPLL (Table 6). The mean opening angles for C4 and C5 did not differ significantly between the CSM and OPLL groups.

Axial pain was reported by $34 \%$ of the patients, including 12 of the 38 patients with CSM (31.6\%) and 7 of the 18 patients with OPLL (38.9\%). Among the 19 patients who complained of axial pain, 9 patients reported no significant difference in the pain severity between the two sides, 4 patients complained of more severe pain only on the open side, and 6 patients complained of more severe pain only on the hinge side. Fifteen of the 38 pa tients (39.5\%) who underwent surgery for C7 complained of axial pain. Four of the 18 patients (22.2\%) who underwent surgery for C6 complained of axial pain. Post-

Table 4. Result of C2 - C7 angles.

\begin{tabular}{cccc}
\hline & CSM & OPLL & P \\
\hline Preop. & $12.7 \pm 12.71$ & $10.9 \pm 7.19$ & NS \\
Postop. 6 mo. & $11.3 \pm 13.82$ & $8.2 \pm 9.37$ & NS \\
Postop. final. & $10.5 \pm 14.23$ & $5.6 \pm 7.23$ & NS \\
\hline
\end{tabular}

NS indicates not significant.

Table 5. Result of ROM between C2 - C7.

\begin{tabular}{cccc}
\hline & CSM & OPLL & P \\
\hline Preop. & $31.5 \pm 12.14$ & $24.6 \pm 9.19$ & NS \\
Postop. 6 mo. & $19.9 \pm 8.71$ & $15.5 \pm 9.88$ & P $<0.05$ \\
Postop. final. & $21.2 \pm 9.25$ & $14.0 \pm 7.99$ & $\mathrm{P}<0.01$ \\
\hline
\end{tabular}

NS indicates not significant.

Table 6. Result of opening angle at C4, C5.

\begin{tabular}{cccc}
\hline & CSM & OPLL & P \\
\hline C4 & $65.9 \pm 5.92$ & $63.5 \pm 6.88$ & NS \\
C5 & $64.8 \pm 8.14$ & $61.8 \pm 5.55$ & NS \\
\hline
\end{tabular}

NS indicates not significant. operative C5 palsy developed on the open side in 1 of the 56 patients $(1.8 \%)$.

\section{Discussion}

Expansive open-door laminoplasty is one of the most common procedures for cervical spondylosis and OPLL, and various modified surgical procedures have been reported. We have developed a unique modified procedure involving placement of a stay suture to ensure that the vertebral arch remains expanded and to avoid complications that can result from bone grafts or spacers.

The clinical outcomes of the modified procedure were satisfactory and were similar to those reported by other studies. The rate of JOA score improvement was about $60 \%$, the lordosis angle observed on lateral radiographs was maintained, and ROM decreased in both the CSM and OPLL groups [9-11].

Our technique for modified expansive open-door laminoplasty has two important characteristics. First, the most caudal part and lower part of the dilated spinous processes are tied by a crank-shaped suture, a simple method to prevent reclosure of the expanded vertebral arch. Second, to keep the vertebral arch expanded, a stay suture is fixed on the fascia at the hinge side, $2 \mathrm{~cm}$ from the center. This holds the vertebral arch up, even in cases with a floating lamina, and prevents the vertebral arch from becoming separated inadvertently. The mean opening angle, as measured on the postoperative CT, was $62^{\circ}$ $65^{\circ}$, and sufficient decompression was obtained.

The suture used to tie the vertebral arches to maintain them in the expanded state was fixed on the fascia. However, 19 of the 56 patients (34\%) complained of axial pain, although the laterality was not clear. However, the pain was not severe enough in any patient to necessitate habitual trigger point or other forms of therapy. The decompression range was set recently as C3 - C6 in general, and a tendency toward a decrease in axial pain has been observed [13-15]. Procedures such as the Shiraishi method, which saves the posterior elements, have been reported to prevent axial pain [16-19].

Postoperative C5 palsy is thought to be caused by trauma induced by the surgical technique, displacement of the lamina on the hinge side, a tethering effect induced by an excessive posterior shift of the spinal cord after decompression, traction stress on the nerve root, or damage to the gray matter of the spinal cord. However, the precise mechanism responsible for C5 palsy remains unclear $[20,21]$. The reported incidence of C5 palsy is about 5\% [22]. In this series, 1 of the 56 patients developed palsy on the open side, and the incidence of this palsy in patients undergoing our modified procedure was low, 1.8\%. This may be explained by the small mean opening angle of the vertebral arch, about $60^{\circ}$, in this procedure, which may prevent the clinical condition caused by traction stress 
or other factors.

\section{Conclusion}

The results of this study show that our modified procedure produces satisfactory postoperative outcomes based on the clinical data and imaging findings for patients with CSM or OPLL. An advantage of this procedure is that it avoids potential complications associated with bone grafts or implants. Moreover, it is as easy to perform as the original procedure and can effectively maintain the vertebral arch in an expanded state.

\section{REFERENCES}

[1] K. Hirabayashi, "Expansive Open-Door Laminoplasty for Cervical Spondylotic Myelopathy,” Shujutu, Vol. 32, No. 11, 1978, pp. 1159-1163.

[2] K. Hirabayashi, K. Watanabe, K. Wakano, N. Suzuki, K. Satomi and Y. Ishii, "Expansive Open-Door Laminoplasty for Cervical Spinal Stenotic Myelopathy,” Spine, Vol. 8, No. 7, 1983, pp. 693-699. doi:10.1097/00007632-198310000-00003

[3] K. Hirabayashi, Y. Toyama and K. Chiba, "Expansive Laminoplasty for Myelopathy in Ossification of the Longitudinal Ligament,” Clinical Orthopaedics and Related Research, Vol. 359, 1999, pp. 35-48. doi:10.1097/00003086-199902000-00005

[4] H. Baba, N. Furusawa, Q. Chen and S. Imura, "Cervical Laminoplasty in Patients with Ossification of the Posterior Longitudinal Ligaments,” Paraplegia, Vol. 33, No. 1, 1995, pp. 25-29. doi:10.1038/sc.1995.6

[5] E. Frank and T. L. Keenen, "A Technique for Cervical Laminoplasty Using Miniplates,” British Journal Neurosurgery, Vol. 8, No. 2, 1994, pp. 197-199. doi:10.3109/02688699409027967

[6] T. Itoh and H. Tsuji, “Technical Improvements and Results of Laminoplasty for Compressive Myelopathy in the Cervical Spine," Spine, Vol. 10, No. 8, 1985, pp. 729736. doi:10.1097/00007632-198510000-00007

[7] Y. Kawaguchi, M. Kanamori, H. Ishihara, K. Ohmori, H. Nakamura and T. Kimura, "Minimum 10-Year FollowUp after En Bloc Cervical Laminoplasty,” Clinical Orthopaedics and Related Research, Vol. 411, 2003, pp. 129139. doi:10.1097/01.blo.0000069889.31220.62

[8] M. F. O’Brien, D. Peterson, A. T. Casey and H. A. Crockard, "A Novel Technique for Laminoplasty Augmentation of Spinal Canal Area Using Titanium Miniplate Stabilization,” Spine, Vol. 21, No. 4, 1996, pp. 474-484. doi:10.1097/00007632-199602150-00012

[9] N. Tanaka, K. Nakanishi, Y. Fujimoto, H. Sasaki, N. Kamei, T. Hamasaki, K. Yamada, R. Yamamoto, T. Nakamae and M. Ochi, "Expansive Laminoplasty for Cervical Myelopathy with Interconnected Porous Calcium Hydroxyapatite Ceramic Spacers: Comparison with Autogenous Bone Spacers,” Journal of Spinal Disorders \& Techniques, Vol. 21, No. 8, 2008, pp. 547-552.

[10] H. Tsuji, "Laminoplasty for Patients with Compressive
Myelopathy Due to So-Called Spinal Stenosis in Cervical and Thoracic Regions,” Spine, Vol. 7, No. 1, 1982, pp. 2834. doi:10.1097/00007632-198200710-00002

[11] N. Tsuzuki, R. Abe, K. Saiki and T. Iizuka, “TensionBand Laminoplasty of the Cervical Spine,” Intanational Orthopaedics, Vol. 20, No. 5, 1996, pp.275-284.

[12] J. Mochida, T. Nomura, M. Chiba, K. Nishimura and E. Toh, "Modified Expansive Open-Door Laminoplasty in Cervical Myelopathy,” Journal Spinal Disorders, Vol. 12, No. 5, 1999, pp. 386-391.

[13] N. Hosono, H. Sakaura, Y. Mukai, R. Fujii and H. Yoshikawa, "C3-6 Laminoplasty Takes over C3-7 Laminoplasty with Significantly Lower Incidence of Axial Neck Pain,” European Spine Journal, Vol. 15, No. 9, 2006, pp. 1375-1379. doi:10.1007/s00586-006-0089-9

[14] H. Sakaura, N. Hosono, Y. Mukai, M. Iwasaki and H. Yoshikawa, "Medium-Term Outcomes of C3-6 Laminoplasty for Cervical Myelopathy: A Prospective Study with a Minimum 5-Year Follow-Up,” European Spine Journal, Vol. 20, No. 6, 2011, pp. 928-933. doi:10.1007/s00586-011-1690-0

[15] T. Takeuchi and Y. Shono, "Importance of Preserving the C7 Spinous Process and Attached Nuchal Ligament in French-Door Laminoplasty to Reduce Postoperative Axial Symptoms," European Spine Journal, Vol. 16, No. 9, 2007, pp. 1417-1422. doi:10.1007/s00586-007-0352-8

[16] T. Shiraishi, "Skip Laminectomy-A New Treatment for Cervical Spondylotic Myelopathy, Preserving Bilateral Muscular Attachments to the Spinous Processes: A Preliminary Report,” Spine Journal, Vol. 2, No. 2, 2002, pp. 108115. doi:10.1016/S1529-9430(01)00118-8

[17] M. Yoshida, T. Tamaki, M. Kawakami, N. Nakatani, M. Ando, H. Yamada and N. Hayashi, "Does Reconstruction of Posterior Ligamentous Complex with Extensor Musculature Decrease Axial Symptoms after Cervical Laminoplasty?” Spine, Vol. 27, No. 13, 2002, pp. 1414-1418. doi:10.1097/00007632-200207010-00008

[18] N. Hosono, H. Sakaura, Y. Mukai, T. Ishii and H. Yoshikawa, "En Bloc Laminoplasty without Dissection of Paraspinal Muscles,” Journal of Neurosurgery, Vol. 3, No. 1, 2005, pp. 29-33. doi:10.3171/spi.2005.3.1.0029

[19] S. Nakama, K. Nitanai, Y. Oohashi, T. Endo and Y. Hoshino, "Cervical Muscle Strength after Laminoplasty," Journal of Orthopaedic Science, Vol. 8, No. 1, 2003, pp. 3640. doi:10.1007/s007760300006

[20] K. Chiba, Y. Toyama, M. Matsumoto, H. Maruiwa, M. Watanabe and K. Hirabayashi, "Segmental Motor Paralysis after Expansive Open-Door Laminoplasty,” Spine, Vol. 27, No. 19, 2002, pp. 2108-2115. doi:10.1097/00007632-200210010-00006

[21] K. Satomi, J. Ogawa, Y. Ishii and K. Hirabayashi, "ShortTerm Complications and Long-Term Results of Expansive Open-Door Laminoplasty for Cervical Stenotic Myelopathy,” The Spine Journal, Vol. 1, No. 1, 2001, pp. 2630. doi:10.1016/S1529-9430(01)00008-0

[22] H. Sakaura, N. Hosono, Y. Mukai, T. Ishii and H. Yoshikawa, "C5 Palsy after Decompression Surgery for Cervical Myelopathy,” Spine, Vol. 28, No. 21, 2003, pp. $2447-$ 2451. doi:10.1097/01.BRS.0000090833.96168.3F 